\title{
Image of the month: Aortic coarctation assessed by contemporary multimodality cardiac imaging
}

\author{
Authors: Bartosz Olechowski, ${ }^{\mathrm{A}}$ Samantha Fitzsimmons, ${ }^{\mathrm{B}}$ Sue Thomas, ${ }^{\mathrm{C}}$ Trevor Richens ${ }^{\mathrm{D}}$ and Peter O' Kane $^{\mathrm{E}}$
}
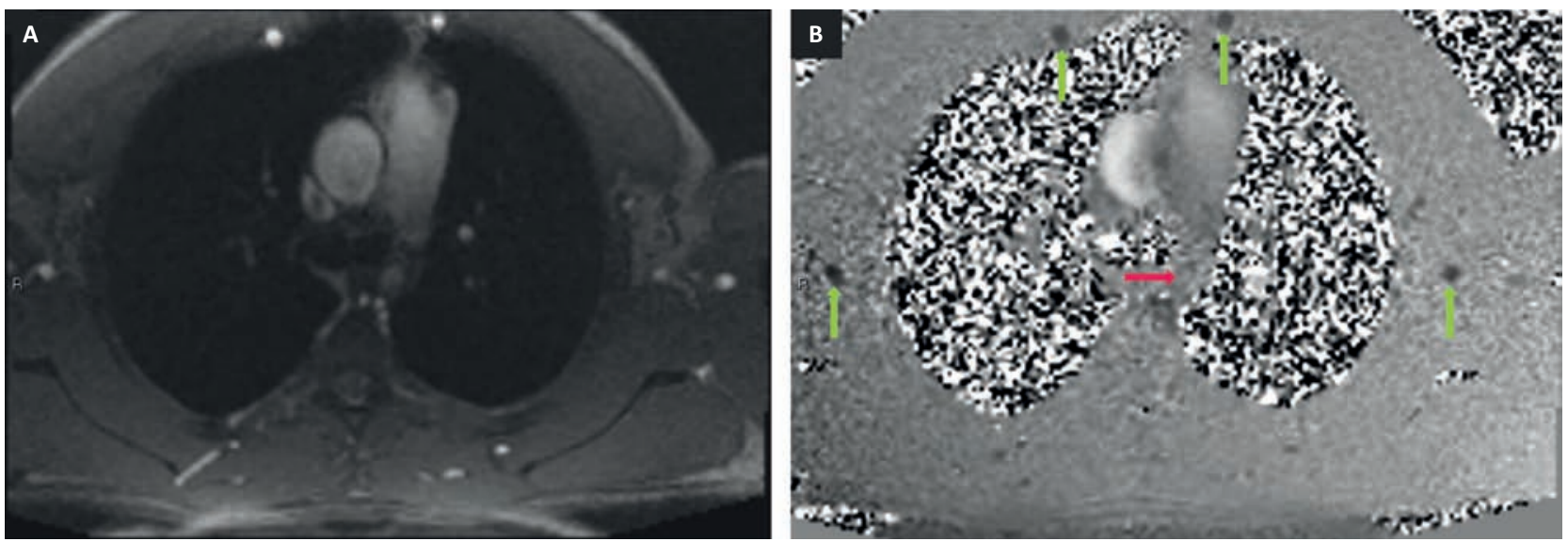

Fig 1. Axial phase contrast thoracic magnetic resonance imaging at the level of the pulmonary trunk. Anatomical magnitude images (A) and velocity encoded images (B) show no detectable flow in the descending thoracic aorta (red arrow) and flow in dilated intercostal and internal mammary collaterals (green arrows).

Hypertension is a common and well recognised clinical entity, which in a majority of cases (95\%) is essential in nature. ${ }^{1}$ In a relatively small proportion of patients, secondary causes of hypertension are found. High blood pressure (BP) may be the first presentation of an undiagnosed congenital coarctation of the aorta, which is a narrowing of the descending aorta typically located at the ligamentum arteriosum just distal to the left subclavian artery.

A 24-year-old man presented with exertional dyspnoea while kickboxing. This led him to duly record his $\mathrm{BP}$ on a home monitor with systolic readings consistently above $180 \mathrm{mmHg}$. He trained intensely 5-6 days per week and described increasing leg cramps during exercise when reviewed in the cardiology clinic several months later. After pharmacological

Authors: ${ }^{\mathrm{A}}$ senior research fellow in cardiology, University Hospital Southampton NHS Trust, Southampton, UK; ${ }^{\mathrm{B}}$ consultant cardiologist, University Hospital Southampton NHS Trust, Southampton, UK; ' Consultant radiologist, The Royal Bournemouth Hospital, Bournemouth, UK; ${ }^{D}$ consultant paediatric cardiologist, University Hospital Southampton NHS Trust, Southampton, UK; ${ }^{E}$ consultant interventional cardiologist, The Royal Bournemouth Hospital, Bournemouth, UK regimens had been initiated, he was referred by his GP for investigation of 'resistant hypertension'.

He had no significant past medical or family history. Clinical examination revealed a 2/6 ejection systolic murmur radiating to the back and evidence of a radial-femoral delay. His BPs were recorded at 155/90 $\mathrm{mmHg}$ (right) and 165/90 $\mathrm{mmHg}$ (left). Electrocardiogram showed left ventricular hypertrophy compatible with longstanding untreated hypertension. Echocardiogram demonstrated an abdominal aorta Doppler trace showing diastolic flows continuation. The descending aorta had a peak velocity of $4.1 \mathrm{~m} / \mathrm{s}$ and maximum velocity of $70 \mathrm{mmHg}$. 24-hour BP monitoring revealed a satisfactory daytime mean of 137/75 mmHg on triple therapy.

Because of his age, symptoms, differential BP reading between each arm and echocardiographic findings, aortic coarctation (CoA) was considered. Thoracic aorta magnetic resonance imaging showed severe CoA immediately distal to the left subclavian artery origin. Flow studies (Fig 1) demonstrated no measurable flow across the coarctation (red arrow) with all descending thoracic aortic flow provided for by large collaterals (green arrows), suggesting a short aortic occlusion. A bicuspid aortic valve and mild annuloaortic ectasia were also noted. Subsequent aortic computerised tomography identified severe coarctation with a patent lumen (3 mm diameter) (Fig 2). The patient was managed 

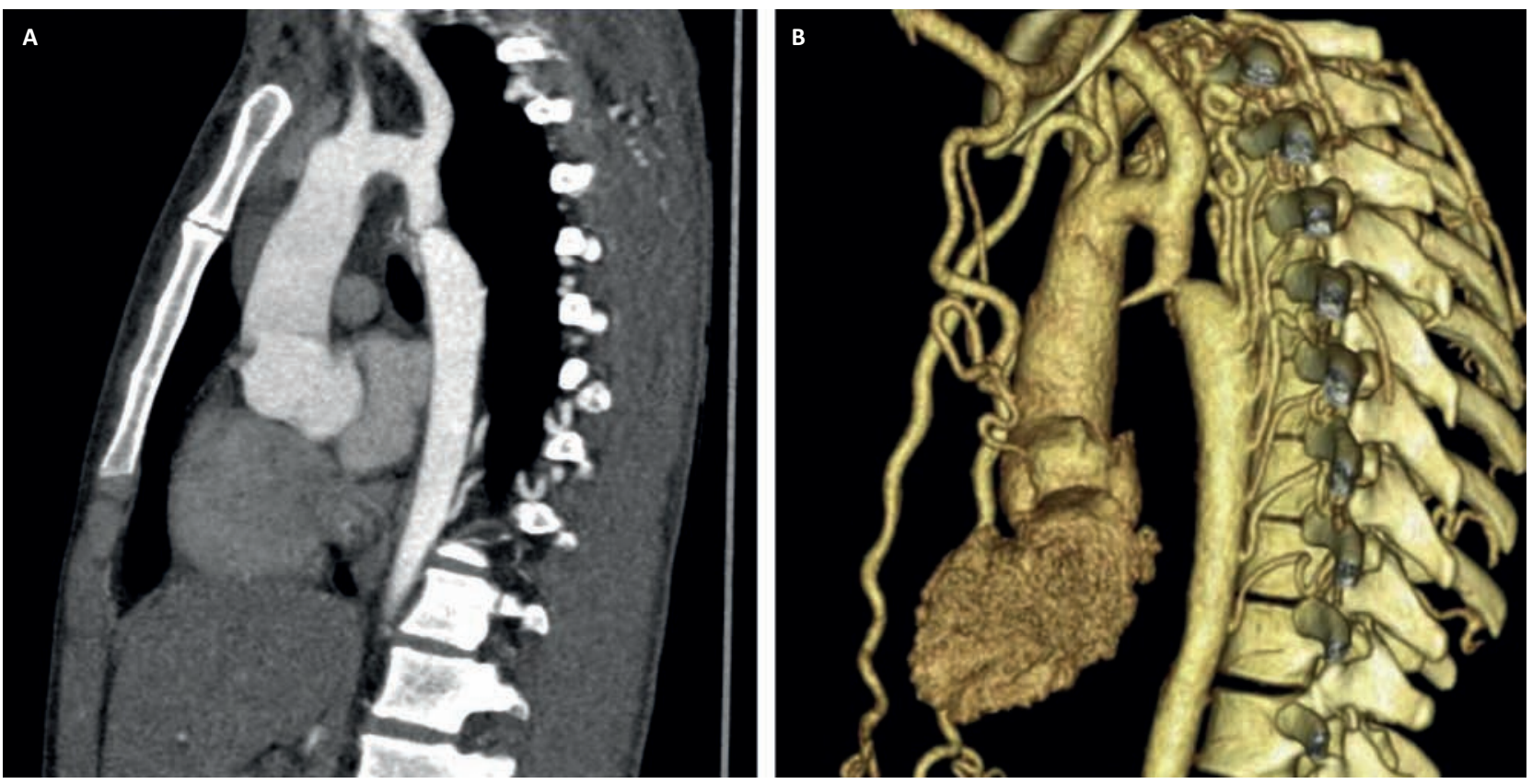

Fig 2. Arterial phase contrast enhanced computerised tomography scan of the thoracic aorta. Sagittal oblique (A) and volume rendered (B) images showing a severe coarctation with a tiny patent lumen.

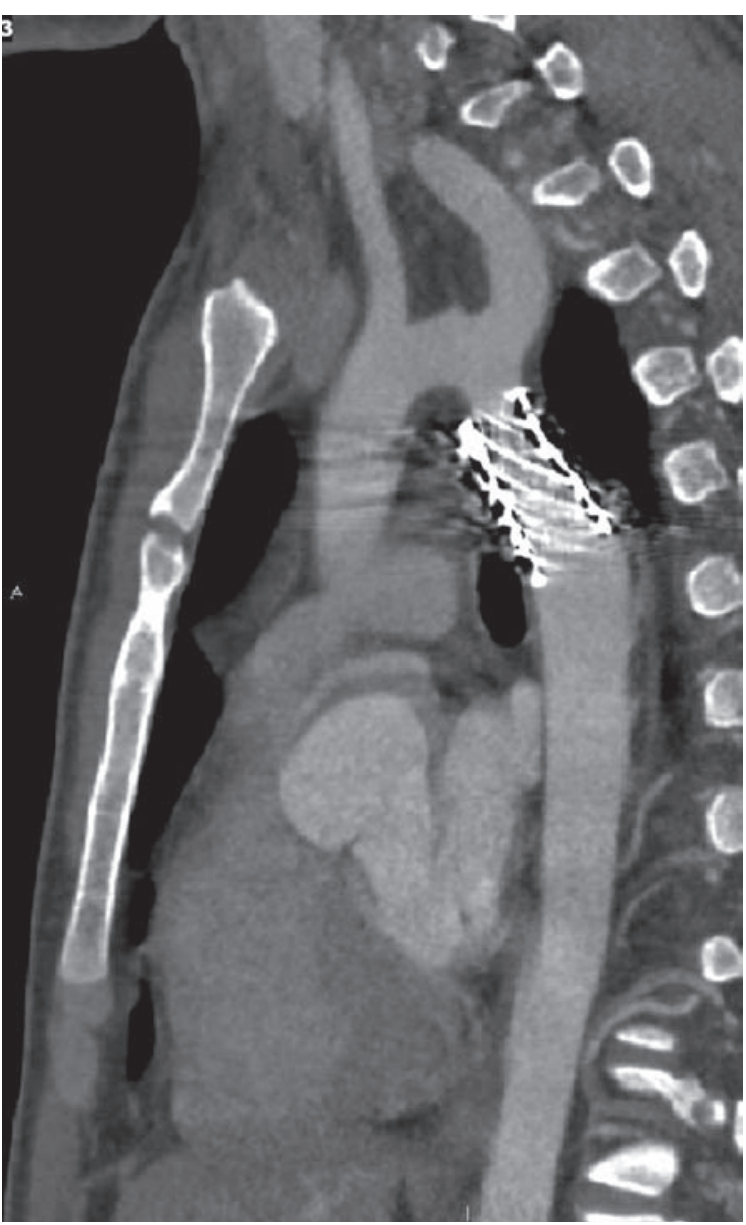

Fig 3. Contrast-enhanced computerised tomography scan showing subsequently stented coarctation. with transcatheter aortic stenting with a Cheatham platinum covered stent (Fig 3).

CoA accounts for approximately $6-8 \%$ of all congenital heart disease and is associated with a bicuspid aortic valve in $20-30 \%$. Systemic hypertension and an increased left ventricular mass are important predictors of cardiovascular mortality and morbidity. ${ }^{2}$ Endovascular stenting is a treatment option in adults with native coarctation, with good outcomes. ${ }^{3}$ This case highlights the importance of contemporary imaging modalities to diagnose and guide interventional therapy for a rare, and often forgotten, cause of a common condition.

\section{Conflicts of interest}

The authors have no conflicts of interests to declare.

\section{Acknowledgements}

Written consent was obtained from the patient to publish the clinical details and images in this article.

\section{References}

1 Carretero OA, Oparil S. Essential hypertension. Part I: definition and etiology. Circulation 2000;25:329-35.

2 De Divitiis M, Pilla C, Kattenhorn M et al. Ambulatory blood pressure, left ventricular mass, and conduit artery function late after successful repair of coarctation of the aorta. J Am Coll Cardiol 2003;41:2259-65.

3 Toro-Salazar OH, Steinberger J, Thomas W et al. Long-term follow-up of patients after coarctation of aorta repair. Am J Cardiol 2002;89:541-5.

Address for correspondence: Dr Bartosz Olechowski,

University Hospital Southampton NHS Trust, Tremona Road, Southampton S016 6YD, UK.

Email: bartosz.olechowski@uhs.nhs.uk 